\title{
EDITORIAL
}

\section{EL ÍNDICE BIBLIOGRÁFICO ESPAÑOL DE CIENCIAS DE LA SALUD. COOPERACIÓN CON LATINOAMÉRICA}

\author{
Jorge Veiga de Cabo \\ Director de la Biblioteca Nacional de Ciencias de la Salud. Escuela Nacional de Sanidad. Instituto de Salud Carlos \\ III. Ministerio de Sanidad y Consumo.
}

En 1950 aparece en EEUU el primer modelo de organización de la politica de investigación. Se crea la National Science Foundation (NSF) con objeto de estructurar las funciones de los organismos implicados en la investigación. Este modelo intenta conservar, aprovechar y rentabilizar las inversiones en infraestructuras tecnológicas y de la industria farmacéutica llevadas a cabo durante la Segunda Guerra Mundial, poniendo a disposición de los núcleos de investigación las tecnologías desarrolladas durante el período bélico, con objeto de fomentar e impulsar la producción científica.

Esta percepción social de la política cientifica, en la que impera la rentabilización de las inversiones y el fomento de la producción científica, se generaliza, imponiéndose como modelo de organización de investigación en la mayoría de los países occidentales. Con el paso del tiempo, los Gobiernos empiezan a plantearse la necesidad introducir criterios de priorización en el modelo, para diseñar la política general de investigación en función de objetivos concretos.

A partir de este momento, las comunidades investigadoras más activas comienzan a trabajar sobre mecanismos que permitan mayor intercambio de información entre los sectores que generan y difunden la producción científica. Se fomentan proyectos que pretenden desarrollar el tercer eslabón de esta cadena de la investigación, el acceso a la documentación. Este planteamiento añade un nuevo elemento a la estructura organizativa anterior y se hace necesario impulsar programas que faciliten la difusión del conocimiento entre y para la comunidad científica que la produce y la demanda en un mismo circuito, en un intento de ordenar la producción en base a criterios de eficacia y eficiencia.

Este marco que parte de un concepto de utilización racional del conocimiento producido, tiene como respuesta inmediata la creación en Estados Unidos, en 1965, de la National Library of Medicine (NLM) como Centro Coordinador de la red de bibliotecas de ciencias de la salud para todo el país. La NLM cuenta en la actualidad con uno de los sistemas más avanzados y completos en tecnologías de comunicación y documentación en ciencias de la salud de los últimos tiempos.

A partir de 1967, y basada en una metodología similar, la Organización Panamericana de Salud, junto con la Organización Mundial de la Salud (OPS/OMS), crean la Biblioteca Regional de Medicina (BIREME), con sede en Sao Paulo (Brasil), que asumirá también la función de Centro Coordinador de la red de bibliotecas de ciencias de la salud de los países latinoamericanos y del Caribe. Ambas instituciones han sido colaboradoras desde el momento 
de su aparición, impulsando el avance tecnológico y desarrollando metodologías que permitan compatibilizar sistemas integrados de comunicación en ciencias de la salud.

Más cercanos a nuestro momento actual y con la aparición de Internet como herramienta y punto de referencia incomparable de sistemas de comunicación, nos encontramos más cerca de poder atender las demandas de la comunidad científica y responder a las necesidades de intercambio y acceso a la documentación. El actual escenario tecnológico y los recursos que nos ofrece es uno de los determinantes del impulso con el que se han visto beneficiados los sistemas compartidos de búsquedas bibliográficas y acceso a la documentación que estamos viviendo en los últimos años. En torno a estos sistemas, y en general a todos los sistemas de interconexión, se ha generado una vertiginosa competencia por mejorar el mantenimiento y puesta al día de la información que se ofrece, que en algunos casos se produce en tiempo real, lo que permite disponer de la más amplia oferta de sistemas de acceso a la información y de difusión del conocimiento.

El concepto de organización de la cadena de difusión del conocimiento biomédico, no ha calado con la fuerza suficiente en nuestro país hasta no hace mucho tiempo, así, en 1996 se crea la Biblioteca Nacional de Ciencias de la Salud (BNCS) que, como Centro del Instituto de Salud Carlos III, y dependiente de la Escuela Nacional de Sanidad, pretende impulsar proyectos que faciliten la gestión entre las bibliotecas de ciencias de la salud y que faciliten la difusión y acceso a la producción científica, tanto de centros de documentación como de la comunidad científica de nuestro país.

El hecho de que España se haya mantenido durante algún tiempo ligeramente al margen de las innovaciones que en este sentido se habían puesto en marcha en otros países, posiblemente lleve aparejado un retraso de nuestra incorporación a las tecnologías apli- cadas a ciencias de la documentación y, aún asumiendo una desventaja de partida, casi con toda seguridad, haya significado una influencia negativa sobre la difusión de publicaciones científicas en nuestra lengua, frente al poderoso mercado anglosajón de publicaciones.

Sin embargo, analizando esta situación desde el punto de vista positivo, quizá parece acertado plantearnos como punto de partida el asumir la necesidad de hacerlo y, como respuesta inmediata, intentar aprovechar las experiencias y la metodologías desarrollada por los países que tienen consolidado el sistema, con las correcciones necesarias para adecuarlo a nuestro entorno sociocultural.

Con este planteamiento y en el marco de un Convenio de Colaboración firmado entre el Instituto de Salud Carlos III con la Organización Panamericana de la Salud/OMS, cuyo objetivo es impulsar acciones conjuntas, uno de los proyectos de cooperación que actualmente se han puesto en marcha por la BNCS y BIREME es el de elaborar una base de datos bibliográfica que incluya las publicaciones del campo de ciencias de la salud publicadas por los países de habla hispana y portuguesa.

Como resultado de la colaboración entre BIREME, la OPS y la OMS, la base de datos LILACS (Literatura latinoamericana y del Caribe), constituye una de las herramientas más importantes para los centros de documentación científica de nuestro entorno, incluyendo referencias bibliográficas de publicaciones latinoamericanas del campo de la biomedicina y de ciencias de la salud. LILACS se encuentra integrada por diferentes áreas del conocimiento, esta disponible en CD-Rom y accesible a través de Internet.

Como una de las principales aportaciones española a este proyecto, la BNCS se encuentra desarrollando el Indice Bibliográfico Español en Ciencias de la Salud (IBECS) que introduce, como innovación respecto a 
otras bases de datos de nuestro entorno más próximo, acoger el mosaico más representativo de publicaciones de diferentes áreas temáticas del campo de las ciencias de la salud, entre las que se encuentran la medicina (incluyendo salud pública, administración sanitaria y especialidades), farmacia, veterinaria odontología, psicologia y enfermería.

Para garantizar un mínimo de calidad en el contenido, recogerá información sobre publicaciones que reúnan una serie de requisitos básicos, en lo que a criterios de rigor científico y editorial se refiere. Para ello, una de las primera acciones que se han llevado a cabo ha sido constituir un Comité Científico que garantice la selección de revistas acorde a parámetros objetivos de valoración y criterios de calidad preestablecidos. El mencionado Comité Científico esta compuesto por representantes de cada uno de los Consejos Generales de las distintas áreas de ciencias de la salud que la componcn. Se cucnta además con un representante de la Federación de Asociaciones Científico-Médicas Españolas (FACME), otro del Centro de Información y Documentación Científica (CINDOC) del Consejo Superior de Investigaciones Científicas (CSIC) y representantes de la propia BNCS. Este Comité Científico se encarga de establecer los criterios de inclusión de las publicaciones que la integren, a la vez que asesorará sobre criterios de calidad durante el desarrollo del mismo.

Uno de los objetivos últimos es la integración de las bases de datos latinoamericana (LILACS) y la española (IBECS) en una base de datos común que sirva de herramienta de utilización conjunta. Para lograr este objetivo el proyecto utilizará lenguajes y metodologías compatibles, lo que a su vez podrá permitir, en posteriores fases del proyecto, compartir también determinados procedimientos de utilidades con protocolos de la red de bibliotecas de la National Library of Medicine de EEUU que ya han sido desarrollados por BIREME.
Por último, este sistema permitirá, además de poder desarrollar y utilizar una base de datos bibliográfica única (LILACS-IBECS), enlazar el proyecto con otros que también se están desarrollando entre la BNCS y BIREME, entre los que podemos destacar: intercambio de información entre bibliotecas de ciencias de la salud españolas con las de la red latinoamericana y del Caribe, mediante sistemas automatizados de búsqueda, gestión $\mathrm{y}$ acceso al documento (proyecto SCAD), desarrollo de la biblioteca virtual de ciencias de la salud (proyecto SciELO), creación del catálogo colectivo de publicaciones periódicas y el análisis de índices de impacto.

Para hacer esto posible, entre las herramientas básicas que incluye la metodología LILACS podemos citar LILDBI, como una aplicación informática desarrollada por BIREME, que ayuda en el proceso de crear los registros bibliográficos de la base de datos LILACS.

LILDBI permite realizar la descripción bibliográfica c indización con la cntrada de todos los datos mínimos y obligatorios que deben ser incluidos en cada registro, así como la asignación a los mismos de Descriptores en Ciencias de la Salud (DeCS) organizados y jerarquizados en categorías (23.000 términos y redes semánticas en ingles, portugués y español). Como herramienta que permitirá compartir aplicaciones con la National $\mathrm{Li}$ brary of Medicine, además de las categorías definidas en el Medical Subject Headings (MeSH), incluye categorías especificas para el área de Salud Pública y forma parte de Unified Medical Language System (UMLS) de la NLM, contribuyendo al desarrollo de sistemas especialistas en lo que se refiere al acceso a fuentes de información.

A través de este proyecto de colaboración entre la BNCS y BIREME, se pretende fortalecer el intercambio de información científica entre Latinoamérica-Caribe y España. Mediante el desarrollo de indices bibliográficos y catálogos colectivos de publicaciones periódicas compartidos, espacios de ac- 
ceso a publicaciones electrónicas en texto completo utilizando tecnología de entorno Internet y contando con un - sitio-Weben la biblioteca virtual SciELO para albergar publicaciones españolas, esperamos poder ayudar a la difusión de las mismas y contribuir a dar soluciones a las principales demandas y necesidades de las bibliotecas de ciencias de la salud, haciéndolos extensivos a toda la comunidad científica española. 